\title{
Comparison of chest drainage tube types (24Fr silastic vs. thoracic catheter) after lung resection
}

Tai Hato ( $\nabla$ taihato@saitama-med.ac.jp )

Saitama Medical Center https://orcid.org/0000-0002-1319-505X

Masatoshi Yamaguchi

Saitama Medical Center

Hiroaki Kashimada

Saitama Medical Center

Ato Sugiyama

Saitama Medical Center

Yoshiaki Inoue

Saitama Medical Center

Kohei Aoki

Saitama Medical Center

Hiroaki Fukuda

Saitama Medical Center

Masathoshi Gika

Saitama Medical Center

Mitsuo Nakayama

Saitama Medical Center

Research article

Keywords: silastic drain, Blake drain, thoracic catheter, lung resection, postoperative drainage

Posted Date: July 14th, 2020

DOI: https://doi.org/10.21203/rs.3.rs-39691/v1

License: (c) (1) This work is licensed under a Creative Commons Attribution 4.0 International License.

Read Full License 


\section{Abstract}

Background: Silastic drains (SDs) are widely used for postoperative chest drainage. We performed a direct comparison of SDs and conventional single-lumen thoracic catheters (TCs) with similar sizes.

Methods: We performed a historically controlled retrospective study. SDs (24 Fr in size) were used for postoperative drainage from September 2017 to May 2018, and TCs (24 or 28Fr) were used from June 2018 to March 2019. Drainage tubes were maintained with a water seal alone. A comparison was performed of the volume and the speed of fluid until $48 \mathrm{hrs}$ after surgery, the undrained area on chest Xray, expansion of subcutaneous emphysema, and the duration of drainage.

Results: One hundred fifty-eight patients with SDs and one hundred fifty-five patients with TCs were included. The patient characteristics were equivalent between the groups. The median length of drainage was two days for both groups. The fluid drainage speed was similar between the groups. While the incidence of expansion of subcutaneous emphysema was equivalent for both groups, reinsertion of drainage tubes was more frequent in the SD group due to insufficient air drainage.

Conclusions: SDs and TCs have almost equivalent drainage performances. For patients with a risk of massive air leakage, SDs should be avoided.

\section{Background}

Silastic drains are already used widely after lung surgery. They have the advantage of comfortability for patients because of their elasticity. Their longitudinal slit length covers a broad area for drainage. Their softness allows flexible tube placement. However, there are potential weaknesses in the unstable tip placement and the potential "bottleneck" of the tube due to the quadrant lumen.

19Fr SDs are popular after lung resection. They are a good alternative to large-bore TCs. Indeed, previous studies compared TCs with 19Fr SDs with almost similar drainage performances. However, continuous aspiration with 19Fr SDs must be applied because of their limited lumen size.

The $24 \mathrm{Fr}$ silastic drain is less popular than the $19 \mathrm{Fr}$ drain. The larger tube size might have some advantages. Continuous aspiration might not be necessary for $24 \mathrm{Fr}$ tubes. Although 24Fr tubes are large, their softness is much better than that of rigid thoracic catheters. The $24 \mathrm{Fr}$ silastic drain is the intermediate between the 19Fr silastic drain and the rigid thoracic catheter.

There have been no previous reports directly comparing the simple structural differences and efficacy of drainage between SDs and TCs. Here, we performed a historically controlled study with these two tubes used in different periods (nine months for both) and compared their outcomes.

\section{Methods}

Study Design 
We performed a historically controlled retrospective study. We used either silastic or thoracic catheters consecutively during the designated period: silastic drain group: September 2017 to May 2018; thoracic catheter group: June 2018 to March 2019. The same team performed all surgeries during these periods.

Patient selection criteria

Consecutive lung resection cases were included. Both thoracotomy and video-assisted thoracic surgery (VATS) cases were included. However, cases of spontaneous pneumothorax were excluded because the duration of drainage was too short.

Drain placement procedure and management

To compare the drainage efficacy, we placed the drainage tube via the same route (Figure 1). We inserted the tube from the "video port" placed in the seventh intercostal space on the posterior axillary line. The drainage tube was placed posterior to the lung, and the tip of the tube was located on the apex of the lung. Drainage tubes were maintained with a water seal alone. Low-pressure aspiration was applied only in patients with expanding subcutaneous emphysema. The drainage tube was removed as soon as possible if the discharge was less than $200 \mathrm{ml} / 24 \mathrm{hr}$ without evident air leakage.

\section{Evaluation}

The amount of drained fluid was obtained from the chart. The fluid was measured hourly on the night of the operation and every eight hours from one day after surgery. The undrained area and subcutaneous emphysema were evaluated by chest X-ray imaging. Chest X-ray images were taken just after the surgery and on days one and two after surgery. Expansion of subcutaneous emphysema and undrained fluid was evaluated by X-ray images.

Statistical analysis

Continuous variables with a normal distribution were compared using Student's t-tests. The MannWhitney test was used to test the significance of the difference when the distribution was not normal. P values less than 0.05 were considered significant.

Ethical declaration

The internal review board approved this study of Saitama Medical Center (No. 2154).

\section{Results}

\section{Patient characteristics}

24Fr SDs were used for postoperative drainage from September 2017 to May 2018, and TCs with a similar size were used from June 2018 to March 2019. One hundred and fifty-eight cases were included in 
the SD group, and 155 cases were included in the TC group. The patient characteristics are summarized in Table 1. There was no significant difference between the groups.

Fluid drainage amounts

The amount of fluid was recorded hourly on the day of the operation and every eight hours from one day after the surgery (Fig. 2 and Table 2). The cases were stratified with or without air leakage. There was no difference between the SD and TC groups regardless of the air leakage throughout the observed periods.

Undrained fluid on chest X-ray

The residual amount of fluid was evaluated by chest X-ray images. Twenty-six patients (16.5\%) had an undrained space on chest X-ray in the SD group, while 31 patients $(20 \%)$ did in the TC group on day two after surgery (Table 3 ). When we focused on lobectomy cases, undrained spaces were more common in left upper lobe cases in the SD group and left lower lobectomy cases in the TC group (Table 4). There was no difference in the right-sided procedures.

Expansion of Subcutaneous emphysema

To compare the drainage efficacy on air, we evaluated subcutaneous emphysema on chest X-ray images on days one and two after surgery. (Table 3) There was no significant difference in terms of incidence between the SD and TC groups. However, reinsertion of the drain was more common in the SD group because of the uncontrollable expansion of subcutaneous emphysema. (Table 5) These four cases of drain reinsertion due to air leakage were not controllable even with the application of continuous aspiration.

\section{Discussion}

Kejriwal and Newman first reported the application of SDs in thoracic surgery [1]. Subsequently, several surgeons reported its use in lung resection with feasible results. [2-5] Currently, 19Fr SDs seem to be more popular than $24 \mathrm{Fr}$ SDs because of their comfortability and less invasiveness. The preceding comparison of SDs with conventional TCs used a 19Fr silastic drain. Whether a silastic catheter is superior to a conventional drain in terms of "structure" has not yet been discussed. Here, we performed a direct comparison of 24Fr SDs with TCs of a similar size to elucidate this issue. Both drains were similarly effective in most of the cases. The incidence of adverse events was almost equivalent, except for the reinsertion of the drainage tube. In the case of massive air leakage, SDs were not effective even with continuous aspiration.

The amount of fluid was almost equivalent, which is compatible with the feasibility study reported by Icard et al.[3] There was a concern about the potential "bottleneck" due to the quadrant lumen structure of SDs. According to the Hagen-Poiseuille equation, the size of the lumen impacts the amount of liquid to the fourth power. $(Q=\pi r 4 / 8 \mu(d p / d x)$, where $Q$ is the volume of the liquid, $r$ is the radius of the lumen, $\mu$ is the viscosity, and $\mathrm{dp} / \mathrm{dx}$ is the pressure gradient). Indeed, in an in vitro experiment using 19Fr SDs 
compared with 28Fr TDs, the fluid drainage capacity was nine times lower with SDs. [6] However, the difference was not meaningful in the in vivo study in terms of the fluid drainage. [6] The result was consistent with the clinical outcomes of our study and other studies. [4, 7]. This is probably because of the slow and/or intermittent fluid flow of pleural effusion.

The drain placement procedure can affect the results. Flexibility is one of the great features of SDs and enables dynamic tube placement. Fukui et al. compared two different placement procedures of the $19 \mathrm{Fr}$ silastic drain. They compared the anterior-to-posterior approach with the posterior-to-anterior approach. [8] They reported that the posterior-to-anterior approach was preferable. Our procedure was similar to the posterior-to-anterior approach. However, the 24Fr SD tube was slightly shorter than the 19Fr silastic drain, which might limit the area of drainage, especially in cases of left upper lobectomy. In the case of left upper lobectomy, the tip of the SD tube could not reach the anterior-cranial area of the residual lobe, which caused residual effusion. Although we selected the posterior-to-anterior approach for all cases this time, it would be much better to change the drain placement route according to the type of surgical procedure.

The incidence of expansion of subcutaneous emphysema was equivalent between the groups. However, there were certain cases of insufficient air drainage. Drain reinsertion was required in four cases due to uncontrollable air leakage. These four cases were managed by reinserting a $28 \mathrm{Fr}$ trocar catheter. This suggests that the drainage tube was the bottleneck of air drainage. Saxena $\mathrm{P}$ et al. also reported their concerns about the air drainage performance of SDs. [9] They reported two cases of insufficient air drainage with SDs after lung resection. From their experience, they reported avoiding the use of SDs in cases of extensive fissure dissection or significant air leakage at the end of the procedure.

Sakakura et al. compared the drainage efficacy of drains with different sizes in experimental models. [7]. They modeled postoperative air leakage using air flow from the respirator to compare the air evacuation pressure and air expulsion time. According to their results, $19 \mathrm{Fr}$ SDs were equivalent to $12 \mathrm{Fr}$ TCs in terms of air drainage. The performance of 24Fr SDs was in between that of 19Fr SDs and $32 \mathrm{Fr}$ TCs. The difference decreased if the expulsion time was shorter. For slow air flow, such as during breathing, the difference was not apparent. However, for fast airflow, such as during coughing, the performance impacted the clinical outcome, such as the expansion of subcutaneous air leakage. When air leakage was substantial, SDs were not suitable for drainage, even with the application of continuous aspiration.

In the case of substantial expansion of subcutaneous emphysema, we applied low-pressure aspiration. However, for most cases, water sealing was sufficient. The four cases of reinsertion in the SD group could not be managed with aspiration. Air drainage and fluid drainage are different. Air flow such as during coughing is much faster and substantial than fluid flow. This means that the structural difference does affect the clinical outcome. However, these cases were exceptional. For most of our cases, air drainage was sufficiently controlled with water sealing. Routine low-pressure aspiration was not required for $24 \mathrm{Fr}$ SDs. 
Two previous reports compared 19Fr SDs with TCs after lung resection. [4, 7] Both reported that 19Fr SDs with aspiration were not inferior to TCs. Our study also showed that 24Fr SDs were almost equivalent to TCs without aspiration in most of the cases. However, it is a burden that SDs cost more than twice the cost of TCs.

Our study has several limitations. First, this study was conducted with a single-center retrospective analysis. The patient backgrounds were almost equivalent between the groups, and a similar number of cases were included. The same surgical team performed all the procedures. Therefore, the allocation of the groups was successful. However, this study was not planned without estimation of statistical power, which might cause obscurity in the interpretation of the results. Second, we inserted the SDs with a similar pathway as TCs. Our intent was simply to compare the performance of SDs with TCs from a structural viewpoint. However, the drainage performance could be affected by the placement procedure. A fixed placement pathway might result in the underestimation of SD performance. Third, we could not collect data on patient comfortability or pain. This might also cause an underestimation of SD performance.

\section{Conclusion}

SDs and TCs with similar sizes showed almost equivalent performances except for in the case of massive air leakage. Careful patient selection and appropriate tube placement procedures would improve the performance of SDs in practice.

\section{Declarations}

Ethical approval and consent to participate

All procedures performed in studies involving human participants were in accordance with the ethical standards of the institutional and national research committee and with the Declaration of Helsinki. The internal review board approved this study of Saitama Medical Center (No. 2154), which waived the requirement for patient consent.

Competing Interests

The authors declare that they have no competing interests.

Consent for publication

Not applicable.

Availability of data and materials

The datasets used and analyzed during the current study are available from the corresponding author on reasonable request. 


\section{Funding}

This study received no funding. All authors declare that there are no financial relationships to disclose regarding this article.

Authors' contribution

TH and MN designed the study. MY, KH, AS, YI, KA, HF, MG, TH and NM collected data. TH and MY analyzed the data. TH, YI and NM wrote the manuscript. All authors read and approved the final manuscript.

Acknowledgements

None.

Authors' information

Affiliations: Department of General Thoracic Surgery, Saitama Medical Center, Saitama Medical University

Address: 1981 Kamoda, Kawagoe, Saitama, Japan 350-8550

TEL/FAX: +81-49-228-3459 /+81-49-228-3459

E-mail; taihato@saitama-med.ac.jp

Tai Hato, Masatoshi Yamaguchi, Hiroaki Kashimada, Ato Sugiyama, Yoshiaki Inoue, Kohei Aoki, Hiroki Fukuda, Masatoshi Gika, Mitsuo Nakayma.

\section{Abbreviations}

SD

Silastic drain

TC

Thoracic catheter

VATS

Video assisted thoracic surgery

\section{References}

1. Kejriwal NK, Newman MA. Use of a single silastic chest drain following thoracotomy: initial evaluation. ANZ J Surg. 2005;75(8):710-2.

2. Ishikura $\mathrm{H}$, Kimura $\mathrm{S}$. The use of flexible silastic drains after chest surgery: novel thoracic drainage. Ann Thorac Surg. 2006;81(1):331-3. 
3. Icard P, Chautard J, Zhang X, Juanico M, Bichi S, Lerochais JP, Flais F. A single 24F Blake drain after wedge resection or lobectomy: a study on 100 consecutive cases. Eur $\mathrm{J}$ Cardiothorac Surg. 2006;30(4):649-51.

4. Nakamura H, Taniguchi Y, Miwa K, Adachi Y, Fujioka S, Haruki T. The use of Blake drains following general thoracic surgery: is it an acceptable option? Interact Cardiovasc Thorac Surg. 2009;8(1):5861.

5. Yamaguchi M, Yoshino I, Kameyama T, Osoegawa A, Tagawa T, Yohena T, Maehara Y. Use of smallbore silastic drains in general thoracic surgery. Ann Thorac Cardiovasc Surg. 2007;13(3):156-8.

6. Niinami H, Tabata M, Takeuchi Y, Umezu M. Experimental assessment of the drainage capacity of small silastic chest drains. Asian Cardiovasc Thorac Ann. 2006;14(3):223-6.

7. Sakakura N, Fukui T, Mori S, Hatooka S, Yokoi K, Mitsudomi T. Fluid drainage and air evacuation characteristics of Blake and conventional drains used after pulmonary resection. Ann Thorac Surg. 2009;87(5):1539-45.

8. Fukui T, Sakakura N, Kobayashi R, Katayama T, Ito S, Hatooka S, Mitsudomi T. Comparison of methods for placing and managing a silastic drain after pulmonary resection. Interact Cardiovasc Thorac Surg. 2009;9(4):645-8.

9. Saxena P, Kejriwal N, Newman MA. Use of flexible silastic drains in thoracic surgery: a word of caution. Ann Thorac Surg. 2007;83(6):2258-9. author reply 2259.

\section{Tables}

Due to technical limitations, the tables can only be accessed as a download in the supplementary files section.

\section{Figures}




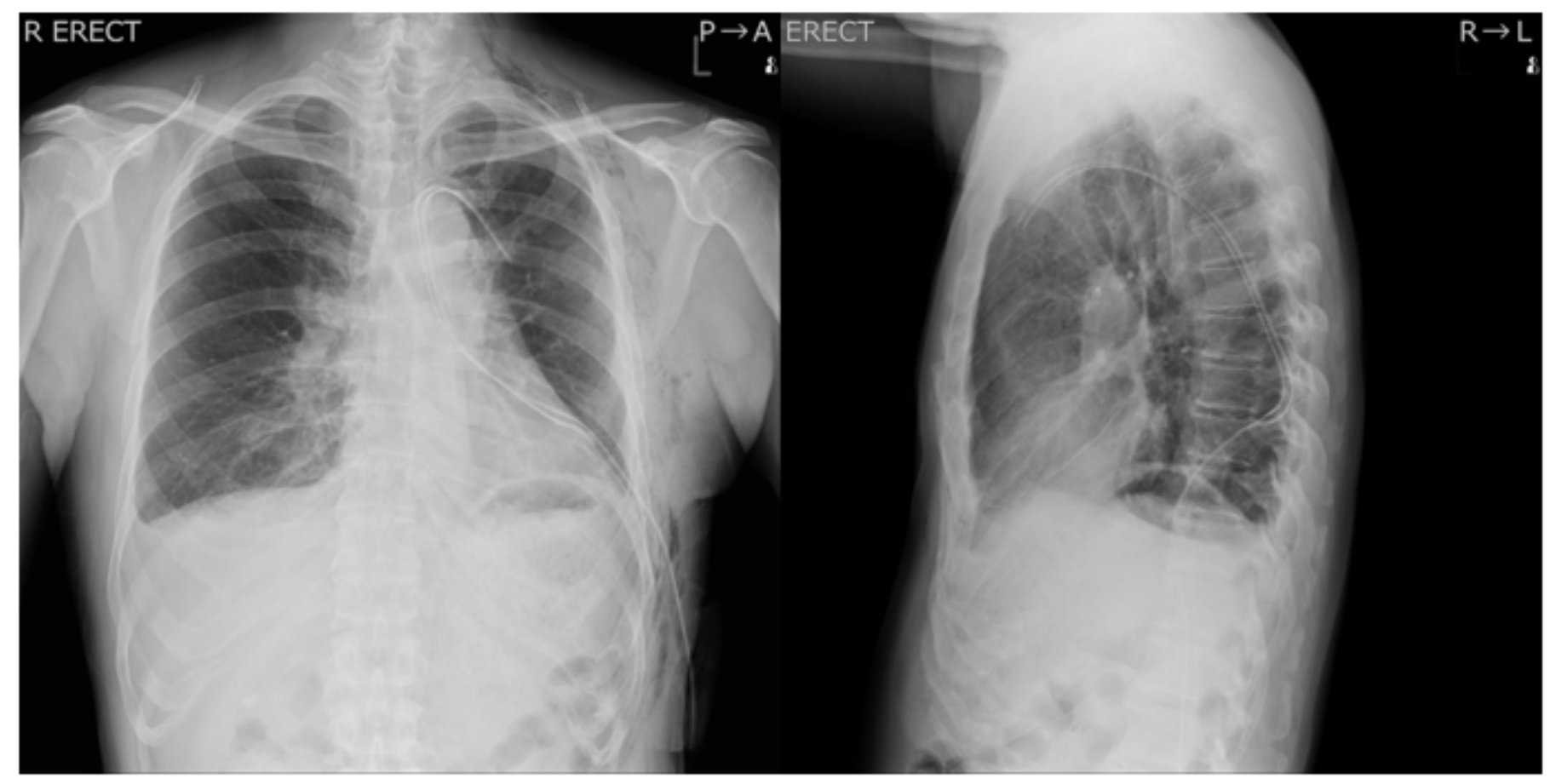

\section{Figure 1}

Representative chest X-ray of the SD tube in this study The X-ray was taken on day two after surgery. The drain was inserted via a posterior-to-anterior approach.

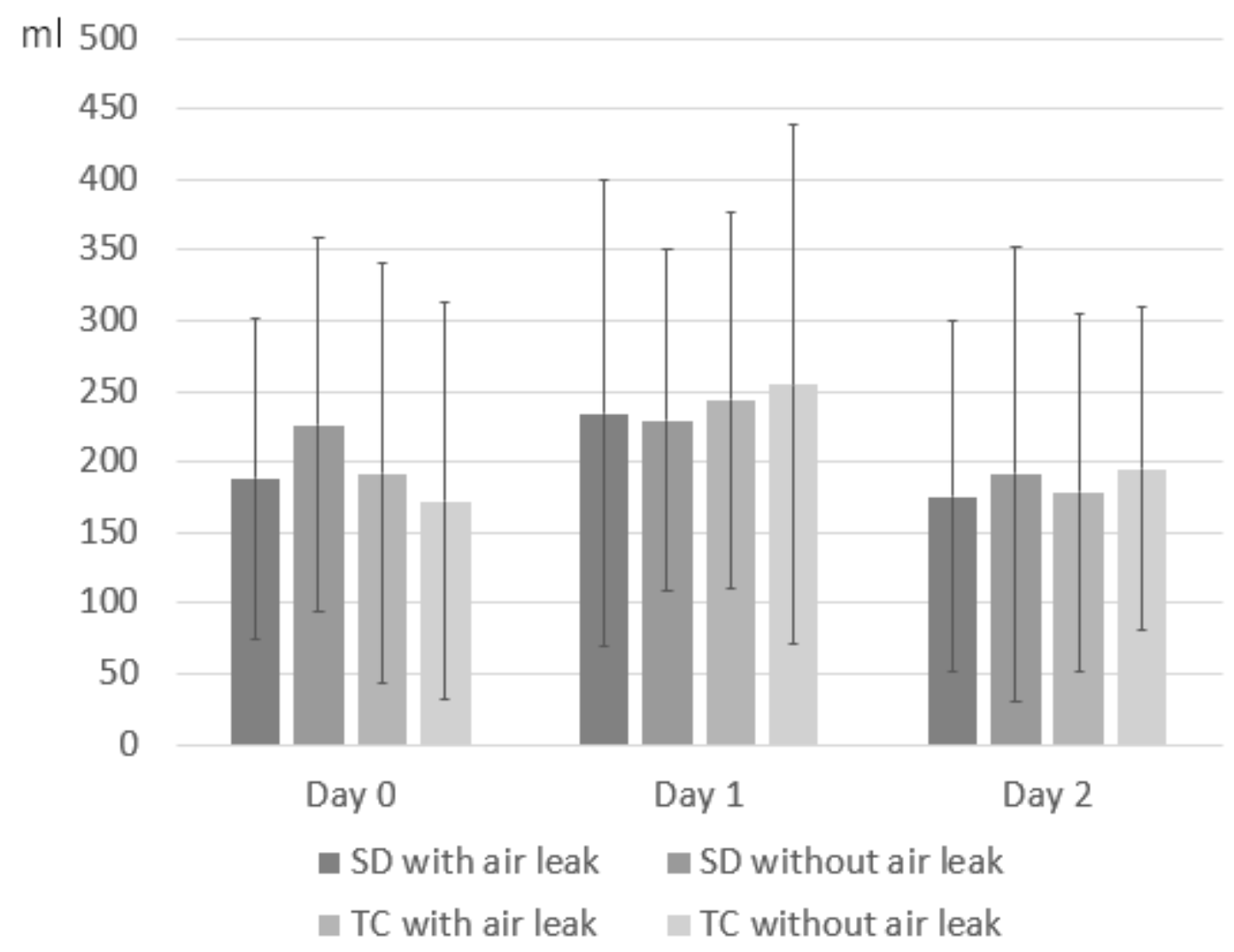

Figure 2 
Amount of drainage fluid at each time point The fluid amount was almost equivalent between the groups, regardless of the presence of postoperative air leakage.

\section{Supplementary Files}

This is a list of supplementary files associated with this preprint. Click to download.

- FiguresandTablesdrainBMCsurg.pptx 